\title{
Overcommitment but not Effort-Reward Imbalance Relates to Stress-Induced Coagulation Changes in Teachers
}

\author{
Roland von Känel, M.D. • Silja Bellingrath, Ph.D. • \\ Brigitte M. Kudielka, Ph.D.
}

Published online: 30 January 2009

(C) The Society of Behavioral Medicine 2009

\begin{abstract}
Background Stress-related hypercoagulability might link job stress with atherosclerosis.

Purpose This paper aims to study whether overcommitment, effort-reward imbalance, and the overcommitment by effort-reward imbalance interaction relate to an exaggerated procoagulant stress response.

Methods We assessed job stress in 52 healthy teachers ( $49 \pm 8$ years, $63 \%$ women) at study entry and, after a mean follow-up of $21 \pm 4$ months, when they underwent an acute psychosocial stressor and had coagulation measures determined in plasma. In order to increase the reliability of job stress measures, entry and follow-up scores of overcommitment and of effort-reward imbalance were added up to total scores.

Results During recovery from stress, elevated overcommitment correlated with D-dimer increase and with smaller fibrinogen decrease. In contrast, overcommitment was not associated with coagulation changes from pre-stress to immediately post-stress. Effort-reward imbalance and the interaction between overcommitment and effort-reward
\end{abstract}

R. von Känel

Division of Psychosomatic Medicine,

Bern University Hospital (Inselspital) and University of Bern,

Bern, Switzerland

S. Bellingrath • B. M. Kudielka $(\bowtie)$

Jacobs Center on Lifelong Learning and Institutional

Development, Jacobs University Bremen,

Campus Ring 1,

28759 Bremen, Germany

e-mail: b.kudielka@jacobs-university.de

S. Bellingrath · B. M. Kudielka

Department of Theoretical and Clinical Psychobiology, Graduate School of Psychobiology, University of Trier,

Trier, Germany imbalance did not correlate with stress-induced changes in coagulation measures.

Conclusions Overcommitment predicted acute stress-induced hypercoagulability, particularly during the recovery period.

Keywords Blood coagulation - Cardiovascular disease . Effort-reward imbalance $\cdot$ Fibrinogen fibrin degradation products $\cdot$ Job stress $\cdot$ Psychological stress

\section{Introduction}

Numerous prospective studies have demonstrated an increased risk of coronary heart disease in individuals who experience chronic stress at work [1-3]. This link seems stronger with cumulative exposure to job strain and is partially independent of established cardiovascular risk factors and adverse health behavior clustering with high job strain in their own right [4]. The pathophysiological mechanisms by which job stress promotes atherosclerosis are not fully understood [2]. One promising line of research suggests that work stress is associated with chronic lowgrade hypercoagulability [5]. A hypercoagulable state of the blood promotes atherosclerosis development and, after rupture of an atherosclerotic plaque, thrombotic occlusion of a coronary artery $[6,7]$.

The job stress model of effort-reward imbalance posits that an imbalance between high efforts and low rewards perceived at the work place leads to sustained strain [8]. In the effort-reward imbalance model, the inability to withdraw from work obligations combined with a high need for approval was conceptualized as a personality trait called overcommitment. Highly overcommitted workers tend to repeatedly exaggerate their efforts at work while, at the 
same time, exhausting their resources. As a consequence, high overcommitment diminishes the potential to recover from job demands, eventually resulting in poor mental and physical health [9], including clinical manifestations of coronary heart disease [8]. Conceptually, effort-reward imbalance and overcommitment might contribute to coronary heart disease risk in three ways [8, 10]: (1) the combination of high efforts with low rewards increases the risk, (2) a high level of overcommitment increases the risk, and (3) the interaction between high effort-reward imbalance and overcommitment bears an even higher risk than high effort-reward imbalance or high overcommitment alone. A study on more than 11,000 Dutch employees showing that the risk of effort-reward imbalance to aggravate physical and psychological complaints was greater in workers with high overcommitment relative to those with low overcommitment supports the latter reasoning [11].

Plasma fibrinogen levels were particularly found to be elevated in epidemiological studies across different working populations and conceptualizations of job stress [5, 12]. Specifically, elevated effort-reward imbalance and overcommitment scores have both been associated with elevated fibrinogen levels at rest [13]. Overcommitment has also been associated with increased plasma levels of type 1 plasminogen activator inhibitor and decreased activity of tissue-type plasminogen activator at rest, both indicating impaired fibrinolysis [14]. However, effort-reward imbalance and overcommitment showed no association with resting level of fibrinogen in another study [15]. Although this has not previously been studied in relation to job stress, different types of chronic psychosocial stressors were shown to elicit an increase in the hypercoagulability marker fibrin D-dimer [16]. Fibrinogen and D-dimer are closely linked within the coagulation cascade since thrombin converts fibrinogen to fibrin, which is dissolved by the fibrinolytic enzyme plasmin whereby D-dimer is generated [17]. Fibrin is the end product of the coagulation cascade and the main component of a blood clot. Therefore, as opposed to individual clotting factors, D-dimer indicates activation of the entire coagulation system [17]. Metaanalyses showed that elevated plasma fibrinogen and Ddimer levels, even within the normal range of the population distribution, predict incident coronary heart disease [18, 19]. Fibrinogen exerts procoagulant function as a precursor of fibrin in the coagulation cascade and as cofactor for platelet aggregation [20].

D-Dimer and fibrinogen are both responsive to acute psychosocial stress [16, 21]. Chronic work stress might contribute to an exaggerated acute procoagulant stress response, thereby promoting atherosclerosis and its thrombotic complications, although such theory has not thoroughly been investigated. One previous study found that men experiencing low job control had greater fibrinogen responses to acute mental stress than men with high job control [22]. In addition, men under high job demands and high blood pressure reactivity in anticipation of a stressor had greater atherosclerosis progression at 4-year follow-up than men with low job demands and low reactivity [23].

We hypothesized that overcommitment, effort-reward imbalance, and the overcommitment by effort-reward imbalance interaction relate to an exaggerated response of plasma D-dimer and fibrinogen levels to acute psychosocial stress in school teachers independent of previous correlates of coagulation measures. We investigated coagulation changes from immediately pre-stress to immediately poststress and also several times during a recovery period of $90 \mathrm{~min}$ after cessation of the stressor. Whether physiological stress responses carry over to the recovery period from stress is of particular clinical importance [24]. Specifically, it is assumed that sustained hypercoagulability in response to acute stress accelerates atherosclerosis progression and growth of a coronary thrombus following plaque rupture and thus myocardial infarction severity [21]. In case of a significant relationship between job stress and stressinduced coagulation changes over time, we therefore performed post hoc analysis to identify whether the effect confines to the immediate stress period, to the recovery period from stress, or to both of these periods.

Stress-induced coagulation changes previously correlated directly and inversely, respectively, with norepinephrine and cortisol reactivity [25]; both were attenuated with high overcommitment [26, 27]. Furthermore, mental health is poor with high job stress [28] and psychological distress (e.g., depression, anxiety, and hostility) was associated with acute stress hypercoagulability [5]. Therefore, we further explored whether stress hormone reactivity and psychopathological symptoms affect the hypothesized relationships.

\section{Materials and Methods}

\section{Study Participants}

Fifty-eight employed school teachers from the region of Trier (Germany) who had previously participated in the first part of the Trier Teacher Stress Study [27, 29] were reinvited between 13 and 27 months after the initial assessment to undergo a follow-up assessment of job stress and a laboratory stress testing. Demographic data, health status, medication, blood pressure, and body mass index were assessed at study entry. Participants with any psychiatric disorder, diabetes, current corticosteroids or psychotropic medication use, or current pregnancy were excluded [29, 30]. Demographic data, health status without body mass index, and medication were also assessed at 
follow-up. For the follow-up investigation, we excluded two smokers, two women taking oral contraceptives, and two participants with missing coagulation measures, leaving a final sample of 52 teachers. The ethics committees of the State Medical Association of Rheinland Pfalz and the University of Trier approved the study protocol. All participants provided written informed consent. They were paid $€ 50$ after completion of the basic study and $€ 70$ after completion of the follow-up stress protocol.

\section{Stress Experiment}

Participants were assessed between 3 and 4 P.M. They were instructed to refrain from physical exercise, a heavy lunch, and alcoholic beverages on the test day. Premenopausal women were tested during the luteal phase of the menstrual cycle to prevent confounding of the procoagulant stress response by female sex hormones [31]. A venous catheter was inserted in the dominant forearm. After a 50-min rest and information about the stress protocol, participants underwent the Trier Social Stress Test consisting of a 3min preparation phase, a 5-min job interview, and a 5-min mental arithmetic task in front of an audience [32]. The Trier Social Stress Test elicits reliable increases in fibrinogen, D-dimer, norepinephrine, and cortisol [21, 25, 32, 33]. After completion of the stress test, participants were instructed to remain seated for $90 \mathrm{~min}$ in a quiet room to recover from the stressor while reading magazines.

\section{Sampling of Blood, Saliva, and Blood Pressure}

Blood for coagulation measures was sampled immediately before, immediately after, and 20, 45, and $90 \mathrm{~min}$ after stress. For the present report, saliva for free cortisol measures was analyzed immediately before stress and 10 , 20, and $30 \mathrm{~min}$ after the stress test. The highest of these values was defined as the peak cortisol response for a particular participant. Blood for norepinephrine measures was collected immediately before and immediately after stress (peak response). Cortisol and norepinephrine reactivity were computed by subtracting pre-stress levels from respective post-stress peak levels. Systolic and diastolic blood pressure was measured immediately before and immediately after the Trier Social Stress Test using an automated device (Omron M5-I, Mannheim, Germany).

\section{Biochemical Analysis}

For the determination of D-dimer and fibrinogen, $3.0 \mathrm{ml}$ of venous blood was added to $0.3 \mathrm{ml}$ of citrate $(0.106 \mathrm{~mol} / \mathrm{L})$ monovettes (Sarstedt, Nümbrecht, Germany). Citrate samples were immediately centrifuged at $4^{\circ} \mathrm{C}$ for $15 \mathrm{~min}$ at $2,000 \times \mathrm{g}$ in an adjacent room. Within $60 \mathrm{~min}$, plasma aliquots were transferred to the core laboratory (Synlab, Trier, Germany) and processed immediately. Fibrinogen was determined by a routine clotting assay following the Clauss method [34] and D-dimer by means of an enzymelinked immunosorbent assay kit (VIDAS ${ }^{\circledR}$ D-dimer Exclusion TM, bioMérieux Marcy-l'Etoile, France). Intra- and inter-assay coefficients of variation were $<4.5 \%$ for fibrinogen and $<6.3 \%$ for D-dimer. Native saliva was collected in 2-ml reaction tubes (Sarstedt, Nümbrecht, Germany) and stored at $-20^{\circ} \mathrm{C}$. Salivary-free cortisol was measured by an in-house time-resolved dissociation-enhanced lanthanide fluorescent immunoassay (intra- and inter-assay coefficients of variation, $<11.6 \%$ ). For norepinephrine measurements, venous blood was sampled in ethylenediaminetetraacetic (EDTA) monovettes and centrifuged for $15 \mathrm{~min}$ at $2,000 \times g$ at $4^{\circ} \mathrm{C}$. Plasma samples were immediately stored at $-80^{\circ} \mathrm{C}$ and analyzed by the core lab by means of high-performance liquid chromatography (intra- and inter-assay coefficients of variation, $<10 \%$ ).

\section{Psychological Assessment}

Effort and reward perceived at work were assessed by the validated German version of the effort-reward imbalance questionnaire [35]. The effort-reward imbalance questionnaire comprises six items related to effort (e.g., "I have a lot of responsibilities in my job"; range, 6-30 points) and 11 items related to reward (e.g., "I receive the respect I deserve from my supervisors/colleagues"; range, 11-55 points). Each item is rated on a five-point Likert scale, whereby the effort and reward scales are equally weighted to compute effort-reward imbalance. A relatively higher effort to reward ratio indicates an unfavorable condition characterized by relatively high effort and relatively low reward. We assessed overcommitment at work using the six-item questionnaire validated in German (range, 6-24 points). On a four-point Likert scale, participants indicate to what extent they agree or disagree with the given statements. Overcommitment focuses on the "inability to withdraw from work" (five items; e.g., "Work is usually still on my mind when I go to bed") and "impatience and disproportionate irritability" (one item). For all three scales, high internal consistencies (Cronbach's alpha) could be demonstrated (effort, $\alpha$ ranging from 0.61 to 0.78 ; reward, $\alpha$ ranging from 0.70 to 0.88 ; and $\mathrm{OC}, \alpha$ ranging from 0.64 to 0.82 ) [36]. In order to increase the reliability of job stress measures, overcommitment and effort-reward imbalance scores at study entry and those at follow-up were added up to a total score of overcommitment and effort-reward imbalance.

Psychopathological symptoms were rated using the revised version of the Symptom Checklist-90 validated in German [37]. In addition to nine syndrome scales (e.g., depression, anxiety, and hostility), not detailed in this paper, 
the sum of all 90 items provides a Global Severity Index. The Global Severity Index is a widely used rating for general psychological distress and level of psychopathologic symptoms, respectively [38].

\section{Statistical Analysis}

Data were analyzed using the Statistical Package for Social Sciences version 15.0 for Windows (SPSS Inc., Chicago, IL) with significance level at $p<.05$ (two-tailed). All data showed a normal distribution as verified by the KolmogorovSmirnov test, except D-dimer and cortisol measures, which were logarithmically transformed before analysis. Independent $t$ tests and chi-square tests were used to estimate group differences in continuous and categorical variables, respectively. Pearson correlation analysis was applied to estimate the bivariate correlation coefficient between two variables.

We applied repeated measures analysis of variance to investigate whether stress-induced changes in D-dimer and fibrinogen levels over time (i.e. "time effect" between immediately pre-stress, immediately post-stress, and 20, 45, and $90 \mathrm{~min}$ post-stress) were associated with continuous values of overcommitment, effort-reward imbalance, and the interaction overcommitment by effort-reward imbalance. Repeated measures analysis of covariance was applied to investigate whether these relationships would be independent of covariates. We selected four control variables a priori to prevent model overfitting [39], namely, pre-stress levels of coagulation measures, gender, age (on performing the Trier Social Stress Test), and body mass index (obtained at study entry). While the first control variable accounted for individual differences in pre-stress levels that might lead to floor or ceiling effects in the magnitude of the procoagulant response, the latter three were previously shown to be correlates of fibrinogen and D-dimer [15, 16]. The Huynh-Feldt correction for the degrees of freedom was applied whenever the sphericity assumption was violated. Effect sizes are expressed as partial eta squared $\left(\eta^{2}\right)$. Post hoc testing applied partial correlation analysis to identify a significant relationship between job stress measures and the magnitude of change between individual time points. Fisher Z-transform was used to investigate whether the strength of two correlation coefficients would significantly differ from each other [40].

\section{Results}

Participant Characteristics

Table 1 presents the characteristics of the 52 teachers studied. Age, years in job, body mass index, and blood pressure measurements refer to data obtained at study entry.
Variables are compared between participants grouped according to a median split on total overcommitment and effort-reward imbalance scores (i.e., scores at study entry plus scores at follow-up). Compared to participants with low overcommitment (14-30 points), those with high overcommitment (32-45 points) had a higher Global Severity Index and lower pre-stress salivary cortisol. In addition, there was a trend towards statistical significance for relatively higher pre-stress norepinephrine levels in the high overcommitment group. Participants with high effortreward imbalance (1.25-4.31) expectedly differed from those with low effort-reward imbalance $(0.47-1.20)$ in terms of all psychological scales.

\section{Change in Job Stress Variables from Study Entry} to Follow-up

Overcommitment $(15.7 \pm 4.6$ vs. $15.1 \pm 4.4 ; p=0.15)$, effort $(15.7 \pm 5.5$ vs. $15.5 \pm 4.5 ; p=0.72)$, reward $(44.1 \pm 8.5$ vs. $45.1 \pm 7.7 ; p=0.22)$, and effort-reward imbalance $(0.72 \pm$ 0.45 vs. $0.67 \pm 0.31 ; p=0.32$ ) did not significantly change between study entry and follow-up. The test-retest correlations were $r=0.78$ for overcommitment, $r=0.71$ for effort, $r=0.75$ for reward, and $r=0.63$ for effort-reward imbalance (all $p$ values $<0.001$ ). This suggests that perceived job stress and the extent to which participants feel committed to their job were stable over time.

Relationship Between Job Stress and Coagulation Measures Before Stress

There were no significant bivariate relationships between overcommitment, effort-reward imbalance, and the overcommitment by effort-reward imbalance interaction on the one hand and pre-stress levels of D-dimer and fibrinogen on the other (all $p$ values $>0.40$ ). These relationships also remained nonsignificant in partial correlation analyses with covariates gender, age, and body mass index (all $p$ values $>0.16$ ).

\section{Physiological Stress Reactivity}

The Trier Social Stress Test provoked a significant change over time in D-dimer $\left(F_{4,204}=6.03, p<0.001 ; \eta^{2}=0.106\right.$; Fig. 1a) and in fibrinogen $\left(F_{3.66,186.76}=4.91, p=0.001 ; \eta^{2}=\right.$ 0.088 ; Fig. 1b). The stress test also provoked increases in systolic blood pressure $(128 \pm 18$ vs. $146 \pm 21 \mathrm{mmHg} ; p<$ 0.001 ), diastolic blood pressure ( $83 \pm 10$ vs. $92 \pm 12 \mathrm{mmHg}$; $p<0.001$ ), and norepinephrine ( $478 \pm 153$ vs. $731 \pm 196 \mathrm{pg} /$ $\mathrm{ml} ; p<0.001)$ from pre-stress to immediately post-stress. Free saliva cortisol increased between immediately prestress and 10,20 , or 30 min post-stress $(3.8 \pm 3.3$ vs. $7.7 \pm$ $4.8 \mathrm{nmol} / 1 ; p<0.001)$. Cortisol and norepinephrine reactivity correlated with each other $(r=0.36, p=0.010)$. Over- 
Table 1 Characteristics of participants and as per overcommitment and ERI category

\begin{tabular}{|c|c|c|c|c|c|c|c|}
\hline & $\begin{array}{l}\text { All } \\
(n=52)\end{array}$ & $\begin{array}{l}\text { High total } \\
\text { OC }(n=26)\end{array}$ & $\begin{array}{l}\text { Low total } \\
\text { OC }(n=26)\end{array}$ & $P$ value & $\begin{array}{l}\text { High total } \\
\text { ERI }(n=25)\end{array}$ & $\begin{array}{l}\text { Low total } \\
\text { ERI }(n=27)\end{array}$ & $P$ value \\
\hline Women/men (\%) & $63 / 37$ & $69 / 31$ & $58 / 42$ & 0.388 & $76 / 25$ & $52 / 48$ & 0.071 \\
\hline Age (years) & $49 \pm 8$ & $50 \pm 8$ & $49 \pm 9$ & 0.832 & $49 \pm 7$ & $50 \pm 9$ & 0.596 \\
\hline Years in job & $24 \pm 10$ & $24 \pm 9$ & $23 \pm 12$ & 0.671 & $22 \pm 10$ & $25 \pm 11$ & 0.229 \\
\hline BMI $\left(\mathrm{kg} / \mathrm{m}^{2}\right)$ & $25.8 \pm 4.7$ & $26.4 \pm 5.9$ & $25.2 \pm 3.2$ & 0.363 & $26.6 \pm 6.0$ & $25.1 \pm 3.0$ & 0.279 \\
\hline SBP (mmHg) & $129 \pm 17$ & $129 \pm 13$ & $129 \pm 21$ & 0.994 & $127 \pm 14$ & $132 \pm 19$ & 0.304 \\
\hline DBP (mmHg) & $83 \pm 10$ & $83 \pm 9$ & $82 \pm 12$ & 0.675 & $83 \pm 10$ & $82 \pm 11$ & 0.883 \\
\hline Total OC & $30.8 \pm 8.5$ & $37.8 \pm 4.2$ & $23.8 \pm 5.1$ & $<0.001$ & $35.6 \pm 6.6$ & $26.3 \pm 7.6$ & $<0.001$ \\
\hline Total effort & $31.2 \pm 9.3$ & $37.5 \pm 6.6$ & $24.9 \pm 7.0$ & $<0.001$ & $38.4 \pm 6.5$ & $24.6 \pm 5.8$ & $<0.001$ \\
\hline Total reward & $89.3 \pm 15.2$ & $82.5 \pm 15.6$ & $96.0 \pm 11.6$ & 0.001 & $77.6 \pm 13.5$ & $100.1 \pm 5.9$ & $<0.001$ \\
\hline Total ERI & $1.40 \pm 0.68$ & $1.80 \pm 0.67$ & $1.00 \pm 0.39$ & $<0.001$ & $1.93 \pm 0.61$ & $0.91 \pm 0.22$ & $<0.001$ \\
\hline GSI & $0.46 \pm 0.39$ & $0.60 \pm 0.43$ & $0.31 \pm 0.28$ & 0.006 & $0.66 \pm 0.43$ & $0.27 \pm 0.22$ & $<0.001$ \\
\hline Follow up (days) & $627 \pm 113$ & $611 \pm 114$ & $644 \pm 112$ & 0.299 & $616 \pm 116$ & $637 \pm 112$ & 0.510 \\
\hline $\mathrm{NE}(\mathrm{pg} / \mathrm{ml})$ & $478 \pm 153$ & $517 \pm 150$ & $437 \pm 148$ & 0.060 & $493 \pm 144$ & $464 \pm 162$ & 0.503 \\
\hline Cortisol (nmol/1) & $3.8 \pm 3.3$ & $2.8 \pm 1.2$ & $4.9 \pm 4.4$ & 0.019 & $3.7 \pm 4.1$ & $3.9 \pm 2.3$ & 0.394 \\
\hline Fibrinogen $(\mathrm{g} / \mathrm{l})$ & $3.1 \pm 1.0$ & $3.2 \pm 1.0$ & $3.0 \pm 0.9$ & 0.270 & $3.0 \pm 1.0$ & $3.2 \pm 1.0$ & 0.430 \\
\hline D-Dimer (ng/ml) & $458 \pm 588$ & $486 \pm 724$ & $431 \pm 423$ & 0.585 & $465 \pm 745$ & $453 \pm 407$ & 0.660 \\
\hline
\end{tabular}

Values are given as mean $\pm \mathrm{SD}$. Concentrations of NE, cortisol, fibrinogen, and D-dimer are pre-stress values and are given in original units $B M I$ body mass index, $D B P$ diastolic blood pressure, ERI effort-reward imbalance, GSI global severity index, $N E$ norepinephrine, $O C$ overcommitment, $S B P$ systolic blood pressure

commitment, effort-reward imbalance, and the overcommitment by effort-reward imbalance interaction did not significantly correlate with stress hormone reactivity up to 30 min after cessation of stress (see also [27]).

Overcommitment and Stress-Induced Coagulation Changes

\section{D-Dimer}

Repeated measures analysis of variance showed a time by overcommitment interaction in predicting stress-related changes in D-dimer levels over time $\left(F_{4,200}=3.05, p=\right.$ $\left.0.018 ; \eta^{2}=0.057\right)$. The interaction was maintained $\left(F_{4,184}=\right.$ $\left.3.38, p=0.011 ; \eta^{2}=0.068\right)$ controlling for gender $(p=0.68)$, age $(p=0.17)$, body mass index $(p=0.049)$, and pre-stress D-dimer levels $(p<0.001)$. Post hoc analysis revealed no significant association between overcommitment and Ddimer increase from pre-stress to immediately post-stress (Table 2). However, overcommitment was associated with greater increases in D-dimer from immediately pre-stress to 45 min post-stress $(p=0.031)$, from immediately post-stress to 45 -min post-stress $(p=0.003)$ and from 20 -min post-
Fig. 1 Coagulation changes over time to acute stress in all participants. The figure shows the significant stress-induced changes between immediately pre-stress (pre-str), immediately post-stress (post-str), 20-, 45-, and 90 -min post-stress in Ddimer (a) and fibrinogen (b). Values are means \pm SEM a Stress-induced change in D-dimer

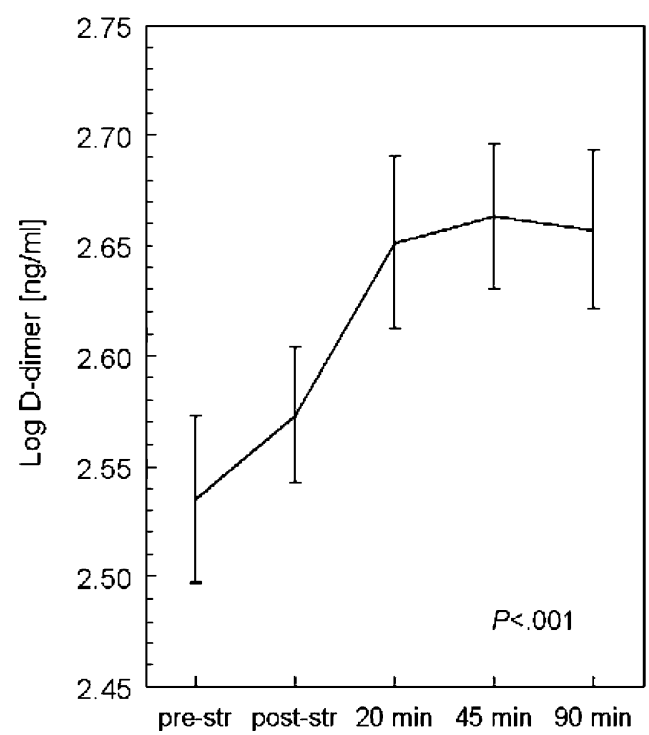

b Stress-induced change in fibrinogen

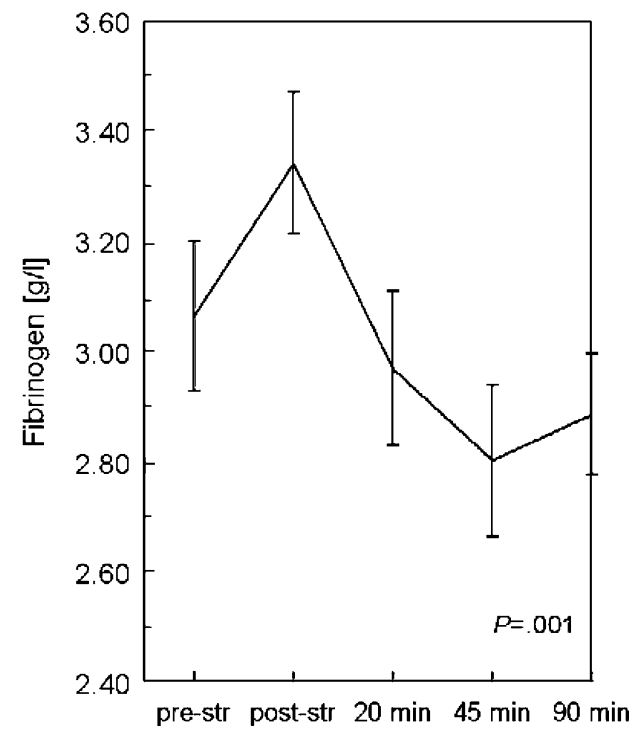


Table 2 Partial correlation between overcommitment and D-dimer changes

Time intervals for change in D-dimer

Partial

correlation

coefficients

Immediately post-stress minus pre-stress

$-0.17$

20-min post-stress minus pre-stress

45-min post-stress minus pre-stress

$-0.18$

$0.31 *$

0.09

90-min post-stress minus pre-stress

$-0.04$

$0.42 * *$

0.19

$0.46^{* * *}$

0.21

0.18

45-min post-stress minus $90-$ min post-stress

Refer to Fig. 1a for the course of D-dimer levels between time points. Correlation coefficients were adjusted for pre-stress D-dimer level, gender, age, and body mass index

$* P \leq 0.05, * * P \leq 0.010, * * * P \leq 0.001$

stress to 45 -min post-stress ( $p=0.001$; Table 2). The strengths of these correlations were not significantly different from each other.

\section{Fibrinogen}

Repeated measures analysis of variance showed a time by overcommitment interaction for stress-related changes in fibrinogen levels over time $\left(F_{3.68,183.89}=2.63, p=0.040\right.$; $\left.\eta^{2}=0.050\right)$. Controlling for gender $(p=0.23)$, age $(p=0.62)$, body mass index $(p=0.43)$, and pre-stress fibrinogen levels $(p=0.001)$, the interaction became marginally significant $\left(F_{3.75,172.47}=2.36, p<0.06 ; \eta^{2}=0.049\right)$. Post hoc analysis showed no relationship between overcommitment and fibrinogen increase from pre-stress to immediately poststress (Table 3). However, there were associations between elevated overcommitment and smaller decreases in fibrinogen from immediately pre-stress to 45 -min post-stress ( $p=$ 0.011 ) and from immediately post-stress to 45 -min poststress $(p=0.042)$. Elevated overcommitment was also associated with a smaller increase in fibrinogen between 45 - and 90-min post-stress $(p=0.004$; Table 3$)$. The strengths of these correlations were not significantly different from each other.

\section{Stress Hormone Reactivity and Psychopathology Symptoms}

We also explored whether controlling for stress hormone reactivity and level of psychopathological symptoms in addition to gender, age, body mass index, and pre-stress levels of the respective coagulation measure would affect the predictive value of overcommitment for stress-induced changes in D-dimer and fibrinogen. However, the signifi-

cances of the time by overcommitment interactions for Ddimer and fibrinogen changes to stress were unaffected when norepinephrine reactivity, cortisol reactivity, and the Global Severity Index were separately added to the models.

Effort-Reward Imbalance and Stress-Induced Coagulation Changes

Effort-reward imbalance did not significantly predict stressinduced changes in D-dimer and fibrinogen levels over time in repeated measures analysis of variance and also not when controlling for gender, age, body mass index, and pre-stress levels of coagulation measures. In addition, controlling for cortisol reactivity, norepinephrine reactivity, and the Global Severity Index did not also change these results.

Overcommitment by Effort-Reward Imbalance Interaction and Stress-Induced Coagulation Changes

Overcommitment did not significantly interact with effortreward imbalance in predicting stress-related changes over time in D-dimer and fibrinogen levels in repeated measures analysis of variance and of covariance with covariates gender, age, body mass index, and pre-stress coagulation measures. These results were maintained when also taking into account stress hormone reactivity and the Global Severity Index.

\section{Discussion}

The primary results of this study suggest that chronic stress at work affects changes in the coagulation measures D-

Table 3 Partial correlation between overcommitment and fibrinogen changes

\begin{tabular}{lc}
\hline Time intervals for change in fibrinogen & $\begin{array}{l}\text { Partial } \\
\text { correlation } \\
\text { coefficients }\end{array}$ \\
\hline Immediately post-stress minus pre-stress & -0.04 \\
Pre-stress minus 20-min post-stress & -0.09 \\
Pre-stress minus 45-min post-stress & $-0.36 *$ \\
Pre-stress minus 90-min post-stress & 0.22 \\
Immediately post-stress minus 20-min post-stress & -0.10 \\
Immediately post-stress minus 45-min post-stress & $-0.30 *$ \\
Immediately post-stress minus 90-min post-stress & 0.13 \\
20-min post-stress minus 45-min post-stress & -0.15 \\
20-min post-stress minus 90-min post-stress & 0.22 \\
90-min post-stress minus 45-min post-stress & $-0.41^{* *}$ \\
\hline
\end{tabular}

Refer to Fig. 1b for the course of fibrinogen levels between time points. Correlation coefficients were adjusted for pre-stress fibrinogen level, gender, age, and body mass index

$* P \leq 0.05, * * P \leq 0.010$ 
dimer and fibrinogen in response to acute psychosocial stress. Specifically, job stress, as measured by the overcommitment and effort-reward imbalance model, was associated with an exaggerated acute procoagulant stress response. We found that elevated levels of overcommitment significantly and independently related to stress-induced fibrin formation as measured by the hypercoagulability marker D-dimer. This relationship was seen in D-dimer changes during the recovery period but not so in D-dimer changes during the immediate stress period. In line with this finding, elevated levels of overcommitment were not associated with the increase in fibrinogen from immediately pre-stress to immediately post-stress but showed associations with a smaller decrease in fibrinogen during the recovery period. The latter was made up by a smaller fibrinogen increase from 45- to 90-min post-stress.

Regardless of overcommitment, we observed that peak levels of D-dimer were reached only 45-min post-stress, whereas fibrinogen levels peaked immediately post-stress to experience a subsequent decline (Fig. 1). The major function of fibrinogen in the coagulation cascade is as the precursor to fibrin, which, in turn, will be split by plasmin, whereby the fibrin degradation produce D-dimer is formed. In other words, only after fibrin has been formed (and fibrinogen has been consumed) will D-dimer be generated; this sequence might explain the different patterns of stressinduced change in D-dimer vs. fibrinogen.

Although not a focus of our study, we did not find an association between overcommitment and effort-reward imbalance and resting levels of coagulation measures. This contrasts with the bulk of previous studies on a relationship between job stress and elevated levels of particularly fibrinogen [5, 12-14]. Limited statistical power could be one explanation as previous studies regularly investigated larger samples.

Effort-reward imbalance did not emerge as a significant predictor of stress-induced coagulation changes in any model. This may suggest that, with regard to job stress, elevated overcommitment is particularly dangerous in terms of procoagulant consequences in response to acute psychosocial stress. We also did not observe a significant time by overcommitment by effort-reward imbalance interaction indicating that the relationship between overcommitment and coagulation changes due to stress exposure was not moderated by effort-reward imbalance. In other words, low effort-reward imbalance will not protect individuals with high overcommitment from experiencing hypercoagulability elicited by acute psychosocial stress. Moreover, the absence of an interaction effect between overcommitment and effort-reward imbalance for stress-induced coagulation changes contrasts theories proposing that workers who experience high levels of both overcommitment and effortreward imbalance are particularly prone to poor health $[8,10]$.
While such an interaction has been observed in relation to poor physical and psychological health [11], only one previous study investigated atherosclerotic outcome [41]. In that study, high levels of overcommitment and effortreward imbalance both predicted incident coronary heart disease, but there was little evidence that effort-reward imbalance was particularly deleterious in individuals with high overcommitment [41]. It should be emphasized that overcommitment refers to a personality trait characterized by enduring cognitive coping strategies with stress $[8,9]$. Therefore, intrinsic effort (i.e., overcommitment) might elicit comparably robust and reliable procoagulant stress responses. In contrast, extrinsic effort-reward imbalance might show greater proneness to subtle fluctuations owing to unpredictable changes in the work environment and, hence, comparably less reliability in terms of a possible relation to stress-induced coagulation changes.

We did not find that stress hormone reactivity and general psychopathology affected the predictive value of job stress for stress-related coagulation changes. Moreover, cortisol and norepinephrine reactivity as well as the Global Severity Index did not emerge as independent predictors of coagulation changes in any model (data not shown). Therefore, overcommitment might adversely impact on cardiovascular health as related to hypercoagulability independent of stress hormone reactivity and poor mental health. For instance, job stress has previously been associated with reduced vagal cardiac control [4], which in turn was demonstrated to relate to hypercoagulability [42]. Future studies may want to investigate whether an exaggerated decrease in vagal tone during acute stress might affect the relationship between overcommitment and coagulation activation.

The positive relationships during recovery time from stress between overcommitment and D-dimer and fibrinogen might be of particular clinical importance. Both exaggerated reactivity of physiological systems to acute mental stress and the incapacity of the biological stress response to recover quickly contribute to somatic diseases [24]. Therefore, hypercoagulability that carries over to the post-stress period might increase the impact on atherosclerosis progression and coronary thrombus formation after plaque rupture more than hypercoagulability rapidly turning off after stress cessation [21]. Given the important role of a hypercoagulable state in general and elevated D-dimer and fibrinogen levels in particular for atherosclerosis and atherothrombotic events, our findings provide one explanation for the increased coronary heart disease risk previously demonstrated in highly overcommitted individuals [41, 4345]. Moreover, our findings might offer an attractive avenue for intervention research. While it has not been investigated whether hypercoagulability can be alleviated by reducing psychosocial stress, cognitive behavioral stress 
management intervention over 3 months previously reduced overcommitment with this effect persisting up to 3 months [46]. Therefore, experimental studies seem warranted focusing on the intrinsic component of job stress in order to perhaps reduce stress procoagulant activity and associated atherothrombotic risk.

The results of our study must be interpreted within the limitations of the study design and population. We investigated healthy, non-smoking, and medication-free teachers who were all employed. This population potentially experiences considerably high job stress [47]. In fact, some participants might have enrolled in the study because they felt highly stressed at work. Our findings might not apply to other working populations and less stressed and unemployed workers. Furthermore, our data and their interpretation may not transfer to patients with manifest cardiovascular diseases and higher prevalence of established cardiovascular risk factors, all of which may affect the magnitude of the procoagulant stress response and its recovery phase [48].

Taken together, we found evidence that elevated overcommitment to work, but not effort-reward imbalance at work, was associated with a hypercoagulable state in response to a standardized acute psychosocial stressor. Overcommitment appeared to elicit sustained coagulation changes to a stressor since hypercoagulability was particularly observed during the recovery period from acute stress. The findings provide one explanation for the increased risk of coronary heart disease in individuals under high job stress.

Acknowledgments This work was supported by Emmy Noether research grant KU 1401/4-1, KU 1401/4-2, and KU 1401/4-3 of the German Research Foundation (DFG) awarded to B.M.K., as well as by the International Research Training Group IRTG funded by the DFG (GRH 1389/1; B.M.K. and S.B. are members of the IRTG).

\section{References}

1. Kivimäki M, Virtanen M, Elovainio M, et al. Work stress in the etiology of coronary heart disease-A meta-analysis. Scand $J$ Environ Health. 2006; 32: 431-442.

2. Rozanski A, Blumenthal JA, Davidson KW, et al. The epidemiology, pathophysiology, and management of psychosocial risk factors in cardiac practice: the emerging field of behavioral cardiology. J Am Coll Cardiol. 2005; 45: 637-651.

3. Kuper H, Marmot M, Hemingway H. Systematic review of prospective cohort studies of psychosocial factors in the etiology and prognosis of coronary heart disease. Semin Vasc Med. 2002; 2: $267-314$.

4. Chandola T, Britton A, Brunner E, et al. Work stress and coronary heart disease: What are the mechanisms? Eur Heart J. 2008; 29: 640-648.

5. von Känel R, Mills PJ, Fainman C, et al. Effects of psychological stress and psychiatric disorders on blood coagulation and fibrinolysis: A biobehavioral pathway to coronary artery disease? Psychosom Med. 2001; 63: 531-544.

6. Falk E, Fernández-Ortiz A. Role of thrombosis in atherosclerosis and its complications. Am J Cardiol. 1995; 75: 3B-11B.

7. Libby P. The molecular mechanisms of the thrombotic complications of atherosclerosis. J Intern Med. 2008; 263: 517-527.

8. van Vegchel N, de Jonge J, Bosma H, et al. Reviewing the effortreward imbalance model: drawing up the balance of 45 empirical studies. Soc Sci Med. 2005; 60: 1117-1131.

9. Siegrist J. A theory of occupational stress. In: Dunham J, ed. Stress in the Workplace: Past, Present and Future. London: Whurr; 2001: 52-66.

10. Tsutsumi A, Kawakami N. A review of empirical studies on the model of effort-reward imbalance at work: Reducing occupational stress by implementing a new theory. Soc Sci Med. 2004; 59: 2335-2359.

11. de Jonge J, Bosma H, Peter R, et al. Job strain, effort-reward imbalance and employee well-being: A large-scale cross-sectional study. Soc Sci Med. 2000; 50: 1317-1327.

12. Theorell T. Job stress and fibrinogen. Eur Heart J. 2002; 23: 1799-1801.

13. Siegrist J, Peter R, Cremer P, Seidel D. Chronic work stress is associated with atherogenic lipids and elevated fibrinogen in middle-aged men. J Intern Med. 1997; 242: 149-156.

14. Vrijkotte TG, van Doornen LJ, de Geus EJ. Work stress and metabolic and hemostatic risk factors. Psychosom Med. 1999; 61: 796-805.

15. Peter R, Alfredsson L, Hammar N, et al. High effort, low reward, and cardiovascular risk factors in employed Swedish men and women: Baseline results from the WOLF Study. $J$ Epidemiol Community Health. 1998; 52: 540-547.

16. von Känel R, Dimsdale JE. Fibrin D-dimer: A marker of psychosocial distress and its implications for research in stressrelated coronary artery disease. Clin Cardiol. 2003; 26: 164-168.

17. Lip GY, Lowe GD. Fibrin D-dimer: a useful marker of thrombogenesis? Clin Sci. 1995; 89: 205-214.

18. Fibrinogen Studies Collaboration. Plasma fibrinogen level and the risk of major cardiovascular diseases and nonvascular mortality: An individual participant meta- analysis. JAMA. 2005; 294: 17991809.

19. Danesh J, Whincup P, Walker M, et al. Fibrin D-dimer and coronary heart disease: Prospective study and meta-analysis. Circulation. 2001; 103: 2323-2327.

20. Koenig W. Fibrin(ogen) in cardiovascular disease: an update. Thromb Haemost. 2003; 89: 601-609.

21. von Känel R, Preckel D, Zgraggen L, et al. The effect of natural habituation on coagulation responses to acute mental stress and recovery in men. Thromb Haemost. 2004; 92: 1327-1335.

22. Steptoe A, Kunz-Ebrecht S, Owen N, et al. Influence of socioeconomic status and job control on plasma fibrinogen responses to acute mental stress. Psychosom Med. 2003; 65: $137-144$.

23. Everson SA, Lynch JW, Chesney MA, et al. Interaction of workplace demands and cardiovascular reactivity in progression of carotid atherosclerosis: population based study. BMJ. 1997; 314: 553-558.

24. McEwen BS. Physiology and neurobiology of stress and adaptation: central role of the brain. Physiol Rev. 2007; 87: 873904.

25. Wirtz PH, Ehlert U, Emini L, et al. The role of stress hormones in the relationship between resting blood pressure and coagulation activity. J Hypertens. 2006; 24: 2409-2416.

26. Wirtz PH, Siegrist J, Rimmele U, et al. Higher overcommitment to work is associated with lower norepinephrine secretion before and after acute psychosocial stress in men. Psychoneuroendocrinology. 2008; 33: 92-99. 
27. Bellingrath S, Kudielka BM. Effort-reward-imbalance and overcommitment are associated with hypothalamus-pituitary-adrenal (HPA) axis responses to acute psychosocial stress in healthy working school teachers. Psychoneuroendocrinology. 2008; 33: $1335-1343$.

28. Kawakami N, Haratani T. Epidemiology of job stress and health in Japan: Review of current evidence and future direction. Ind Health. 1999; 37: 174-186.

29. Bellingrath S, Weigl T, Kudielka BM. Cortisol dysregulation in school teachers in relation to burnout, vital exhaustion, and effortreward-imbalance. Biol Psychol. 2008; 78: 104-113.

30. von Känel R, Bellingrath S, Kudielka BM. Association between burnout and circulating levels of pro- and anti-inflammatory cytokines in school teachers. J Psychosom Res. 2008; 65: 51-59.

31. Jern C, Manhem K, Eriksson E, et al. Hemostatic responses to mental stress during the menstrual cycle. Thromb Haemost. 1991; 66: 614-618.

32. Kudielka BM, Hellhammer DH, Kirschbaum C. Ten years of research with the Trier Social Stress Test (TSST) - Revisited. In: Harmon-Jones E, Winkielman P, eds. Social Neuroscience. New York: Guilford; 2007: 56-83.

33. Dickerson SS, Kemeny ME. Acute stressors and cortisol responses: A theoretical integration and synthesis of laboratory research. Psychol Bull. 2004; 130: 355-391.

34. Clauss A. Gerinnungsphysiologische Schnellmethode zur Bestimmung des Fibrinogens. Acta Haematol. 1957; 17: 237-246.

35. Rödel A, Siegrist J, Hessel A, et al. Fragebogen zur Messung beruflicher Gratifikationskrisen. Zschr Diff Diag Psychol. 2004; 25: $227-238$.

36. Siegrist J, Starke D, Chandola T, et al. The measurement of effortreward imbalance at work: European comparisons. Soc Sci Med. 2004; 58: 1483-1499.

37. Franke GH. SCL-90-R. Die Symptom-Checkliste von Derogatis. 2nd ed. Weinheim: Beltz; 2002.
38. Derogatis L. Symptom Checklist-90-R. Baltimore: Clinical Psychometric Research; 1983.

39. Babyak MA. What you see may not be what you get: A brief, nontechnical introduction to overfitting in regression-type models. Psychosom Med. 2004; 66: 411-421.

40. Papoulis A. Probability and Statistics. New York: Prentice-Hall; 1990.

41. Kuper H, Singh-Manoux A, Siegrist J, et al. When reciprocity fails: Effort-reward imbalance in relation to coronary heart disease and health functioning within the Whitehall II study. Occup Environ Med. 2002; 59: 777-784.

42. von Känel R, Orth-Gomér K. Autonomic function and prothrombotic activity in women after an acute coronary event. $J$ Womens Health. 2008; 17: 1331-1337.

43. Joksimovic L, Siegrist J, Meyer-Hammer M, et al. Overcommitment predicts restenosis after coronary angioplasty in cardiac patients. Int J Behav Med. 1999; 6: 356-369.

44. Peter R, Siegrist J, Hallqvist J, Reuterwall C, Theorell T, SHEEP Study Group. Psychosocial work environment and myocardial infarction: improving risk estimation by combining two complementary job stress models in the SHEEP Study. J Epidemiol Community Health. 2002; 56: 294-300.

45. Siegrist J Peter R, Motz W, Strauer BE. The role of hypertension, left ventricular hypertrophy and psychosocial risks in cardiovascular disease: prospective evidence from blue-collar men. Eur Heart J. 1992; 13Suppl D: 89-95.

46. Aust B, Peter R, Siegrist J. Stress management in bus drivers: A pilot study based on the model of effort-reward imbalance. Int $J$ Stress Manag. 1997; 4: 297-305.

47. Weber A, Weltle D, Lederer P. Ill health and early retirement among school principals in Bavaria. Int Arch Occup Environ Health. 2005; 78: 325-331.

48. von Känel R. Hemostasis and Stress. In: Fink G ed. Encyclopedia of Stress. 2nd ed. Oxford: Elsevier; 2007, volume 2: 300-305. 